\title{
Endoscopic ultrasound-guided Nd:YAG laser ablation of recurrent pancreatic neuroendocrine tumor: a promising revolution?
}
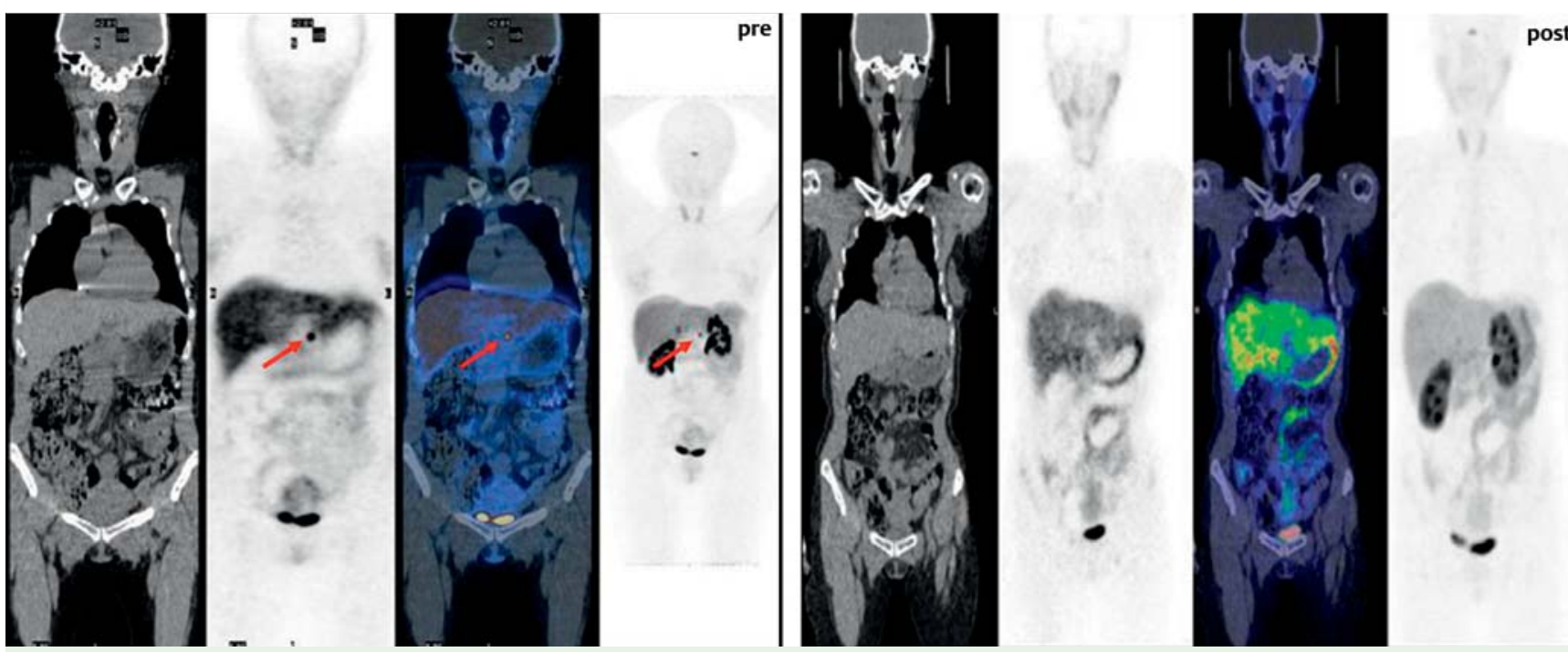

Fig. 1 Pre-treatment ${ }^{68} \mathrm{Ga}$-DOTA-NOC positron emission tomography (PET) revealed a lesion in the residual pancreatic body with metabolic activity (arrow). ${ }^{68} \mathrm{Ga}$-DOTANOC PET 1 year after laser ablation (on the right), showed no metabolic activity in the pancreatic region.
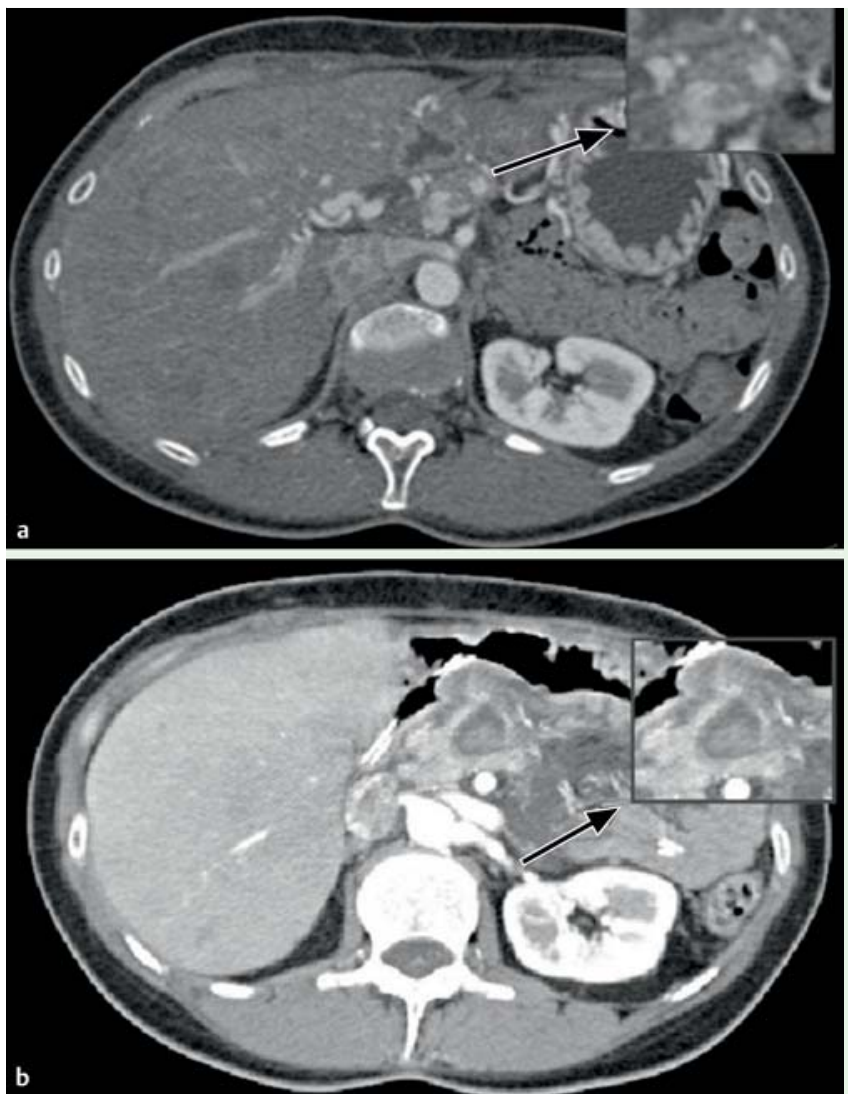

Fig.2 Computed tomography scan. a Recurrent pancreatic neuroendocrine tumor appeared as a 9-mm nodular area with early contrast enhancement on the pancreatic residual body. $\mathbf{b}$ At the same site after treatment, a 35-mm coagulative necrotic area was seen with the onset of a small fluid collection in the omental bursa.
Endoscopic ultrasound (EUS) has developed from a diagnostic tool into a therapeutic one [1]. A 46-year-old woman was diagnosed with recurrent pancreatic neu- roendocrine tumor (PNET) by positive ${ }^{68} \mathrm{Ga}-[1,4,7,10$-tetraazacyclododecane1,4, 7, 10-tetraacetic acid]-1-NaI3-octreotide ( ${ }^{68} \mathrm{Ga}-\mathrm{DOTA}-\mathrm{NOC}$ ) positron emission tomography (PET) ( $\bullet$ Fig. 1 a). Two years earlier she had undergone curative distal pancreatectomy for previous PNET in the setting of multiple endocrine neoplasia type I. At computed tomography (CT) scan, the lesion appeared as a 9-mm nodular area with early contrast enhancement on the pancreatic residual body ( Fig.2a).

The patient refused total pancreatectomy. Laser ablation under EUS guidance was therefore performed using a neodymiumdoped yttrium aluminum garnet (Nd: YAG) laser, at $4.0 \mathrm{~W}$ for 300 seconds (๑ Fig.3). No complications occurred during the procedure. At CT immediately after laser ablation, the ablated lesion appeared as a well-defined 35-mm coagulative necrotics area ( Fig.2b). Neither perilesional parenchymal alteration nor vascular damages were observed. The 2month follow-up CT scan showed the ablated area to be $18 \mathrm{~mm}$ ( $\bullet$ Fig. $4 \mathrm{a}$ ); at 1 year the area was $9 \mathrm{~mm}$ ( $\bullet$ Fig. $4 \mathbf{b}$ ), with no metabolic activity on ${ }^{68} \mathrm{Ga}-D O T A-N O C$ PET ( Fig. 1 b).

Laser ablation is a minimally invasive method for destroying tumors within solid organs, and works by directing lowpower laser light energy into the tissue. It has been used for primary and secondary liver tumors [2-4]. The potential advantage of laser ablation over other laser-in- 

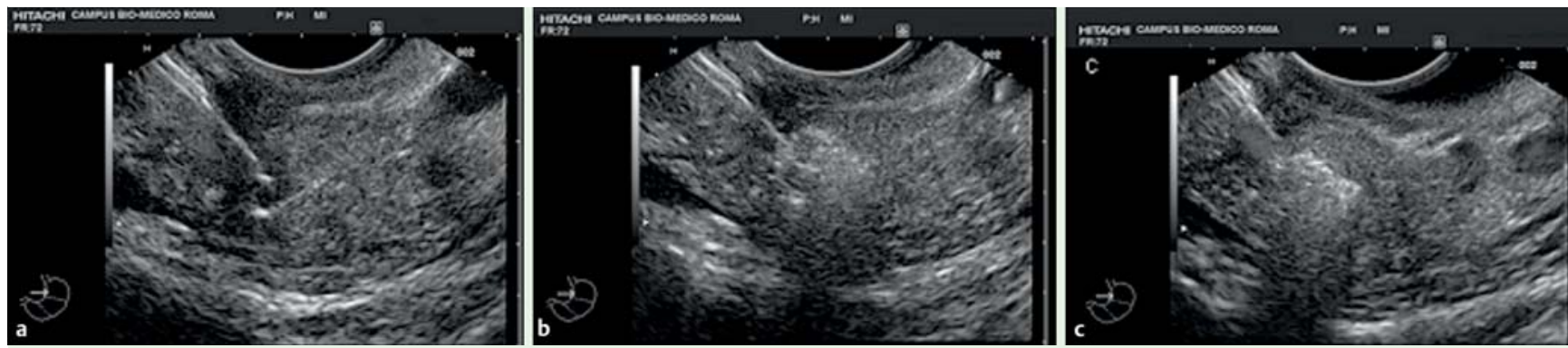

Fig. 3 Endoscopic ultrasound-guided laser ablation. a A hyperechoic line identified the laser fiber protruding from the tip of the needle. $\mathbf{b}$ During laser ablation, a slowly enlarging and coalescing hyperechoic zone appeared around the distal tip of the laser fiber. c After laser ablation, the entire treated area was occupied by an irregular and poorly defined echogenic zone.
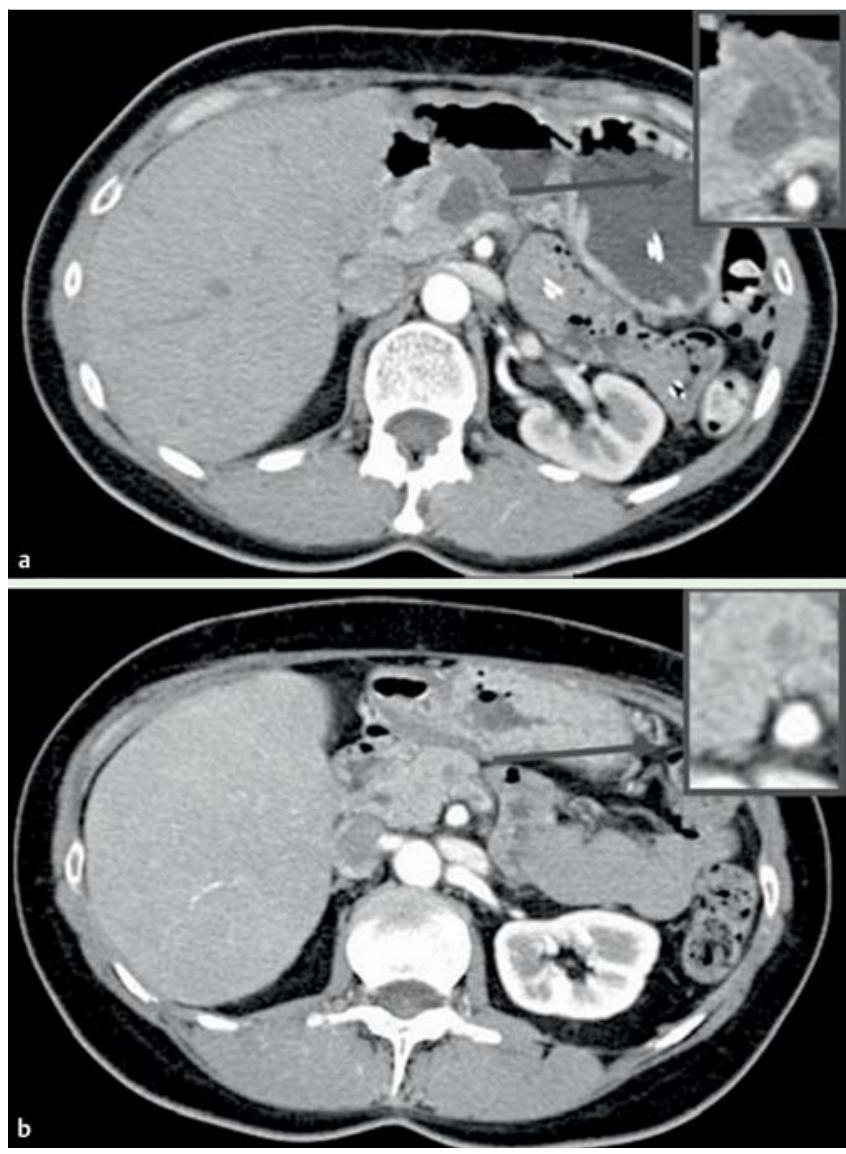

duced thermotherapies is the short application time and the well-defined ablation area. Moreover, the use of thinner laser fibers enables insertion into standard EUS-needles and their potential application in deep abdominal organs, such as the pancreas, which are untreatable using the percutaneous approach. To date, our group has demonstrated the efficacy and safety of laser ablation with Nd:YAG in an in vivo animal model [5]. The current case is the first time we have applied this minimally invasive laser treatment to the human pancreas.

These results must be further assessed in additional patients affected by PNETS who are not suitable for conventional treatments.
Fig. 4 Follow-up computed tomography scans. a At 2 months after laser ablation, the ablated area was further reduced $(18 \mathrm{~mm}$ vs. $35 \mathrm{~mm}$ ). b At 1 year after laser ablation, a 9-mm area of coagulative necrosis remained.$$
\text { tive necrosis remained. }
$$

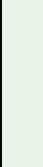

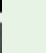

